Preface

\title{
Editorial Compilation VII
}

\author{
Emmanuel J. Favaloro, PhD, FFSc (RCPA) ${ }^{1}$ Giuseppe Lippi, MD ${ }^{2}$ \\ ${ }^{1}$ Department of Haematology, Sydney Centers for Thrombosis and \\ Haemostasis, Institute of Clinical Pathology and Medical Research \\ (ICPMR), Westmead Hospital, Westmead, NSW, Australia \\ 2 Section of Clinical Biochemistry, University of Verona, Verona, Italy \\ Semin Thromb Hemost 2019;45:429-432.
}

Welcome to the latest issue of Seminars in Thrombosis and Hemostasis (STH) published under the "banner" of "Editorial Compilation," this being the seventh such issue. Although STH is primarily a theme-driven publication, ongoing opportunities arise to publish some issues containing more wideranging contributions of current interest and controversy, which do not quite match a current-themed issue in progress. We also require a medium to enable publication of contributions from our Eberhard F. Mammen Young Investigator Award winners. ${ }^{1-8}$ As now standard for this compilation series, the current issue has a mixture of content that comprises such elements, as well as broadly fitting within the separate themes of "thrombosis" and "bleeding."

This issue begins with several contributions related to the arena of preanalytical variables, or laboratory or potentially point-of-care diagnostics. ${ }^{9-11}$ The first article provides some guidance around preanalytical variables, which may affect the results of many routine coagulation and also diagnostic hemostasis assays. ${ }^{12}$ While advances in laboratory instrumentation have partially addressed the laboratory's ability to recognize some of these variables, there remains increased reliance on laboratory personnel to recognize the three potential areas where coagulation testing preanalytical issues may emerge, these being (1) specimen collection (including patient selection), (2) specimen transportation and stability, and (3) specimen processing and storage. The purpose of this article is to identify the preanalytical variables associated with hemostasis testing and provide laboratory practice recommendations in an effort to improve quality of such testing and accuracy of result reporting. ${ }^{9}$

Next is a contribution around the topic of thromboelastography/thromboelastometry (TEG/TEM), in particular its application in the neonatal population. Hemostasis is a dynamic age-related process, which gradually evolves from fetal life, throughout childhood until adulthood. ${ }^{13}$ Although at birth a hemostatic deficit of most coagulation factors is commonplace, some studies have shown that this "hemostatic immaturity" is functionally counterbalanced in healthy term or preterm newborns. Age-related effects around platelet function have also been reported. ${ }^{14}$ The delicate hemostatic balance, however, may be deranged in sick neonates, thus resulting in enhanced risk of hemorrhage and/or thrombosis. In critically ill neonates, conventional coagulation tests may not provide reliable information or indications regarding the functional status of platelets or fibrinolysis, especially in real time. In contrast, viscoelastic tests, namely TEG/TEM, hold promise for rapid assessment of the whole hemostatic potential, allowing immediate intervention should this be required. However, neonatal data are limited due to lack of reference values, especially in premature neonates. In this narrative review, the authors provide some insights around current knowledge regarding TEG/TEM applications in healthy and sick newborns. Overall, the use of viscoelastic tests in the diagnosis and management of coagulation disorders in neonates is worthy of further exploration. The authors further propose that consideration should be made to include these tests in the routine laboratory investigation of neonates, and specific transfusion algorithms should also be developed to avoid treatment pitfalls. $^{10}$

This issue of STH continues the exploration of laboratory testing with a third contribution, by Ames and colleagues, on coagulation activation markers in antiphospholipid syndrome (APS). ${ }^{11}$ In particular, prothrombin fragment F1 + 2 $(\mathrm{F} 1+2)$ and thrombin-antithrombin (TAT) have been assessed in APS, but without evaluating a direct relationship with antiphospholipid (aPL) antibody titers. The authors of this hybrid report, comprising a systematic review and metaanalysis combined with a short report of original data, aimed to investigate whether a direct relationship between aPL and $\mathrm{F} 1+2$ could be identified, as well as to perform a systematic review and meta-analysis of F1 +2 and TAT in APS. The
Address for correspondence Emmanuel J. Favaloro, PhD, FFSc (RCPA), Department of Haematology, Institute of Clinical (RCPA), and Giuseppe Lippi, MD. Pathology and Medical Research (ICPMR), Westmead Hospital, Westmead 2145, Australia (e-mail: emmanuel.favaloro@health. nsw.gov.au).
Issue Theme Editorial

Compilation VII; Guest Editors:

Emmanuel J. Favaloro, PhD, FFS
(RCPA), and Giuseppe Lippi, MD
Copyright @ 2019 by Thieme Medical Publishers, Inc., 333 Seventh Avenue, New York, NY 10001, USA.

Tel: +1(212) 584-4662.
DOI https://doi.org/ 10.1055/s-0039-1692704. ISSN 0094-6176. 
authors performed a systematic search of several databases from January 1982 to December 2018, and then undertook a random effects meta-analysis for continuous outcomes. They also performed an ex vivo study as a cross-sectional casecontrol evaluation of immunoglobulin $\mathrm{G} / \mathrm{immunoglobulin} \mathrm{M}$ $(\mathrm{IgG} / \mathrm{IgM})$ anticardiolipin (aCL) anti- $\beta_{2}$-glycoprotein-I $\left(\mathrm{a} \beta_{2} \mathrm{GPI}\right)$, antiprothrombin (aPT) antibodies, $\mathrm{F} 1+2$ and lupus anticoagulants (LA), as measured in 25 thrombotic primary APS (PAPS), 9 nonthrombotic carriers of aPL, and 18 controls. For the meta-analysis, the significant effect size (ES) for $\mathrm{F} 1+2$ between aPL + ve and aPL - ve SLE and between aPL + ve SLE and control displayed high heterogeneity. The significant ES for F1 +2 between aPL - ve SLE and controls displayed no heterogeneity. The ES for TAT between aPL + ve and aPL - ve SLE patients and between aPL - ve SLE and controls were low, without heterogeneity. For the original study, mean $\mathrm{F} 1+2$ was greater in PAPS $(p \leq 0.0001)$, inversely correlating with IgG aCL, IgM aPT, and LA $(p=0.001,0.03$, and 0.01 , respectively), though only $\operatorname{IgG}$ aCL negatively predicted $\mathrm{F} 1+2(p=0.01)$. The authors concluded that (1) IgG aCL inversely predicts $\mathrm{F} 1+2$; (2) $\operatorname{IgG~aCL}$ positivity introduces heterogeneity in the $\mathrm{F} 1+2 \mathrm{ES}$; and (3) the lack of heterogeneity in the ES for TAT may indicate poor TAT formation in aPL + ve group. Thus, F1 + 2 measurements may be unfounded as already demonstrated for TAT in the 1990s. ${ }^{11}$

Continuing the exploration of APS, Tufano et $\mathrm{al}^{15}$ review its cardiac manifestations, including clinical presentation, role of cardiac imaging, and treatment strategies. As noted earlier, APS is an autoimmune disorder characterized by the presence of aPL, but also vascular thrombosis (venous, arterial, or small vessels) and/or pregnancy morbidity. Diagnosis of APS is based on the presence of at least one clinical criterion (thrombotic events or pregnancy morbidity) and at least one of the laboratory criteria (persistently medium/ high titer IgG/IgM aCL, and/or medium/high titer IgG/IgM $\mathrm{a} \beta_{2} \mathrm{GPI}$, and/or a LA test, confirmed after repetition at least 12 weeks apart. The clinical spectrum of APS encompasses additional (extracriteria) clinical manifestations, including cardiac diseases. Heart involvement may manifest as a consequence of direct (autoimmune-mediated) or indirect (thrombosis) mechanisms, and include heart valve disease (vegetations and/or thickening associated with functional abnormalities) and intracardiac thrombosis, coronary and vascular-accelerated atherosclerosis, along with ischemic heart disease. APS can also cause pulmonary arterial hypertension, left ventricular dysfunction, and heart failure. This review is thus aimed at describing the major cardiac manifestations of APS and illustrating the important role of cardiac imaging for diagnosing subclinical and overt heart involvement and addressing management of these patients. The possible role of therapeutic strategies in cardiac manifestations of APS is also discussed. ${ }^{15}$

The "prothrombosis" theme continues, with several contributions around therapy. ${ }^{16-18}$ First, Christiansen and colleagues report a systematic review and meta-analysis around the topic of primary prevention of cardiovascular events with aspirin, essentially concluding that such treat- ment is heading toward more harm than benefit. ${ }^{16}$ The authors begin by reminding readers that primary prevention of cardiovascular events using aspirin remains controversial, as the risk of bleeding might outweigh the benefits. ${ }^{17} \mathrm{New}$ evidence has recently emerged from ARRIVE (Aspirin to Reduce Risk of Initial Vascular Events), ASCEND (A Study of Cardiovascular Events in Diabetes), and ASPREE (Effect of Aspirin on Cardiovascular Events and Bleeding in the Healthy Elderly) trials. The aims of this report are to provide a systematic review and meta-analysis of aspirin's efficacy and safety in the primary prevention of cardiovascular events in healthy individuals and in those with cardiovascular risk factors, and separately in diabetic patients. The authors searched the Medline database, without time restrictions, for relevant human trials published in English up to December 10,2018, and additional trials were also identified from reference lists. Data on efficacy (cardiovascular death and nonfatal myocardial infarction) and safety (major bleeding) were extracted for analysis. In total, 20 randomized trials were identified. Separate meta-analyses were performed on 10 trials including 144,930 individuals, who were healthy or had cardiovascular risk factors, and on 4 trials including 20,326 individuals with diabetes. In healthy individuals and individuals with cardiovascular risk factors, aspirin reduced the risk of nonfatal myocardial infarction by $21 \%(p<0.001)$, but had no effect on cardiovascular death $(p=0.52)$, and increased the risk of major bleeding by $48 \%(p<0.001)$. In individuals with diabetes, aspirin had no effect on nonfatal myocardial infarction $(p=0.93)$ or cardiovascular death $(p=0.92)$, and enhanced the risk of bleeding by $49 \%$ $(p=0.13)$. This meta-analysis thus suggests that the use of aspirin on routine basis in primary prevention of cardiovascular events seems unadvisable, especially in individuals with diabetes.

A second exploration on thrombosis treatment/prevention is provided by Lippi and colleagues, ${ }^{18}$ who provide a state-of-the-art review on current and emerging direct oral anticoagulants (DOACs). As would be recognized by the readership of this journal, anticoagulant drugs comprise a specific subcategory of antithrombotic agents that act to inhibit blood coagulation at various stages, reducing clot development and ultimately lowering the risk of developing new onset or recurrent thrombosis. Although the long history of anticoagulant drugs has been characteristically shaped by coumarin and heparin derivatives, ${ }^{19-21}$ DOACs represent a new generation of anticoagulants, recently emerged, and that specifically inhibit thrombin or activated factor X. These DOACs combine many advantages of their progenitor drugs, and are therefore prepotently revolutionizing the landscape of antithrombotic therapy. Several drugs (apixaban [BMS-562247], dabigatran [BIBR953], edoxaban [DU-1766], rivaroxaban [BAY 59-7939]) have already received widespread approval by national or supranational medicinal agencies. ${ }^{22,23}$ However, this narrative review primarily explores several other DOACs at different stages of clinical evaluation (betrixaban, darexaban, eribaxaban, letaxaban, and nokxaban), and certain others whose development has been discontinued (AZD-0837, fidexaban, 
LY517717, odiparcil, otamixaban, TTP889, and ximelagatran). What clearly emerges from the authors' analysis is that DOACs sharing very similar mechanisms of action are still characterized by different efficacy and safety profiles. This not only depends on biochemical, biological, and pharmacokinetics characteristics but also on lack of standardization between different clinical trials in terms of targeted disease, patient recruitment, sample size, duration and endpoints, as well as lack of harmonization around procedures used for drug licensing. These factors contribute to challenging the minds of physicians, who may find difficulty navigating their way through multiple indications, different pharmacological profiles, various side effects, and specific drug-to-drug interactions. Such considerations also burden laboratory professionals, who may face organizational and economic challenges in developing and/or implementing multiple assays to assess the pharmacodynamics (effect on coagulation) or pharmacokinetics (drug levels) of DOACs.

DeLoughery and colleagues also discuss emerging treatments for thrombosis. ${ }^{24}$ The authors begin by reminding readers of STH that although anticoagulation without hemorrhage is a primary aim of thrombosis prevention/ treatment, this vision has remained as yet out of reach. Despite the superior safety profile of DOACs, hemorrhage remains a major risk of anticoagulation. Selective inhibition of the contact pathway of coagulation, specifically coagulation factors XI and/or factor XII, has now substantial epidemiologic and preclinical data supporting the notion that these factors contribute to pathologic thrombosis and are yet primarily dispensable for in vivo hemostasis. In this way, targeting factors XI and XII may revolutionize the future anticoagulation landscape. Several drugs are under development for this purpose, including ISIS 416858 , a factor XI antisense oligonucleotide which impairs hepatic synthesis of factor XI; MAA868, a monoclonal antibody that binds the procoagulant enzymatic site of both zymogen and activated factor XI; BAY 1213790, a monoclonal antibody that binds the procoagulant enzymatic site of activated factor XI only; and $\mathrm{AB023}$, a monoclonal antibody that inhibits activated factor XII-mediated activation of factor XI, along with two small molecules in clinical trials. Each of these drugs demonstrated favorable safety profiles in their phase 1 and 2 studies to date, with preclinical data also supporting efficacy of abrogating thrombosis in various animal models. Other benefits of some of these drugs include once-monthly dosing and safety in patients with renal or hepatic impairment, while others offer quickly metabolized parenteral options, thus providing more convenient and widely available anticoagulation options. Though still far from the marketplace, drugs targeting factor XI and factor XII have the potential to usher in a new era of anticoagulation therapy.

We move this issue of STH from purely prothrombotic considerations to other considerations in hemostasis. ${ }^{25-27}$ First, the article by Horstman and colleagues describes the putative role of tissue factor-negative cell-derived microparticles in hemostasis. ${ }^{25}$ Circulating cell-derived microparticles exhibit procoagulant activity and have been investigated for a possible role in some human pathologies.
However, their potential role in hemostasis has been neglected and often denied. This review brings to attention a specific body of direct clinical evidence supporting an important but distinctive role of microparticles in hemostasis. Evidence for a role of microparticles in hemostasis includes (1) two congenital bleeding disorders, attributed to impaired release of microparticles; (2) two recent studies of trauma patients, relating to naturally elevated endogenous microparticles at admission with reduced transfusion requirements and better outcomes; (3) a study of coronary surgery patients, showing that elevated microparticles before surgery reduces transfusion requirements during surgery; and (4) a clinical study of patients with immune thrombocytopenia, demonstrating that patients with high circulating microparticles have reduced bleeding compared with those with similar platelet counts but lower microparticle levels. Mechanisms involving potentiating the contact factor pathway are thought to play a key role and are probably synergistic with polyphosphate released from activated platelets at sites of endothelial injury. The authors conclude that hemostatic defects of patients with deficient microparticle-mediated coagulation resembles deficiency of FXI (i.e., also sometimes known as hemophilia C), distinct from hemophilia A or B; so, it can be termed "type C hemostasis." A better understanding of this proposed hemostatic pathway may lead to improved methods for controlling excessive bleeding in surgery, trauma, and other clinical settings.

Next, de Mello and colleagues performed a systematic review and meta-analysis of hemostatic abnormalities in dementia. ${ }^{26}$ Alzheimer's disease is considered the most frequent cause of dementia. It is known that vascular risk factors play an important role in the development and progression of this condition. Alterations in vascular walls represent documented findings in patients with Alzheimer's disease and other dementias affecting elderly people. The authors aimed to synthesize observational studies that evaluated how the hemostatic system may contribute to cognitive decline in the elderly, using articles published until April 2018 and as indexed in several databases. Among 5,278 studies identified, 33 were included in the final synthesis, and these included 485 patients with mild cognitive impairment (MCI), 588 with vascular dementia, 1,781 with Alzheimer's disease, and 2,895 participants without dementia. Patients with Alzheimer's disease had increased plasma von Willebrand Factor (VWF), plasminogen activator inhibitor-1 (PAI-1), and homocysteine levels. In contrast, the vascular dementia group showed increased fibrinogen levels, activated factor VII, factor VIII, VWF, D-dimer, and homocysteine. The authors conclude by stating that Alzheimer's disease patients show an elevation in some markers of endothelial dysfunction, whereas patients with vascular dementia present mostly an involvement of coagulation cascade components.

The final full-length article in this issue of the journal is by Tabibian and coworkers, who provide a comprehensive overview of coagulation factor $\mathrm{V}(\mathrm{FV})$ and congenital FV deficiency. ${ }^{27}$ Coagulation FV is a glycoprotein with an essential 
role in the formation of the prothrombinase complex, in turn critical for progressing clot formation. FV deficiency is a rare bleeding disorder with an estimated incidence of 1 per 1 million in the general population. The disorder is manifested with a wide array of clinical bleeding events. The most common bleeding features of FV deficiency are mucosal bleedings. Life-threatening manifestations are rarely seen in this disorder. FV deficiency is diagnosed using routine coagulation tests and FV activity assays, supplemented with FV antigen if available. A wide spectrum of mutations including missense, nonsense, and frameshift is observed throughout the F5 gene. Although fresh frozen plasma (FFP) is the dominant therapeutic choice, a newly introduced plasmaderived FV concentrate was found effective in in vitro correction of prothrombin time, activated partial thromboplastin time, and thrombin generation parameters in severe FV deficiency, and should provide more targeted treatment for patients with FV deficiency in the future.

As usual for these nonthematic issues of STH, this issue also contains some correspondence and associated commentaries. ${ }^{28-32}$ We again thank all the authors to this latest issue of "Editorial Compilations" for their original and comprehensive contributions, and we hope our readers enjoy this new installment in this series.

Conflicts of Interest

None declared.

\section{References}

1 Favaloro EJ. 2011 Eberhard F. Mammen award announcements. Semin Thromb Hemost 2011;37(05):431-439

2 Favaloro EJ. 2012 Eberhard F. Mammen award announcements. Semin Thromb Hemost 2012;38(05):425-432

3 Favaloro EJ. 2013 Eberhard F. Mammen award announcements. Semin Thromb Hemost 2013;39(06):567-574

4 Favaloro EJ. 2014 Eberhard F. Mammen award announcements: part II-Young Investigator Awards. Semin Thromb Hemost 2014; 40(07):718-723

5 Favaloro EJ. 2015 Eberhard F. Mammen award announcements: part II-Young Investigator Awards. Semin Thromb Hemost 2015; 41(08):809-815

6 Favaloro EJ. 2016 Eberhard F. Mammen award announcements: part II-Young Investigator Awards. Semin Thromb Hemost 2017; 43(03):235-241

7 Favaloro EJ. 2017 Eberhard F. Mammen award announcements: Part II-Young Investigator Awards. Semin Thromb Hemost 2018; 44(02):81-88

8 Favaloro EJ. 2018 Eberhard F. Mammen award announcements: Part II-Young Investigator Awards. Semin Thromb Hemost 2019; 45(02):123-129

9 Gosselin RC, Marlar RA. Pre-analytical variables in coagulation testing: setting the stage for accurate results. Semin Thromb Hemost 2019;45(05):433-448

10 Konstantinidi A, Sokou R, Parastatidou S, et al. Clinical application of thromboelastography/thromboelastometry (TEG/TEM) in the neonatal population: a narrative review. Semin Thromb Hemost 2019;45(05):449-457

11 Ames PRJ, Bucci T, Iannaccone L, Marottoli V, Arcaro A, Gentile F, Ciampa A. Validity of coagulation activation markers in antiphospholipid syndrome: a systematic review and meta-analysis with a short report. Semin Thromb Hemost 2019;45(05):458-467
12 Lippi G, Favaloro EJ. Preanalytical issues in hemostasis and thrombosis testing. Methods Mol Biol 2017;1646:29-42

13 Favaloro EJ, Lippi G. Translational aspects of developmental hemostasis: infants and children are not miniature adults and even adults may be different. Ann Transl Med 2017;5(10):212

14 Hvas AM, Favaloro EJ. Platelet function testing in pediatric patients. Expert Rev Hematol 2017;10(04):281-288

15 Tufano A, Di Minno MND, Guida A, Lembo M, Di Minno G, Galderisi M. Cardiac manifestations of antiphospholipid syndrome: clinical presentation, role of cardiac imaging, and treatment strategies. Semin Thromb Hemost 2019;45(05):468-477

16 Christiansen M, Grove EL, Hvas A-M. Primary prevention of cardiovascular events with aspirin towards more harm than benefit: a systematic review and meta-analysis. Semin Thromb Hemost 2019;45(05):478-489

17 Lippi G, Danese E, Favaloro EJ. Harms and benefits of using aspirin for primary prevention of cardiovascular disease: a narrative overview. Semin Thromb Hemost 2019;45(02):157-163

18 Lippi G, Gosselin R, Favaloro EJ. Current and emerging direct oral anticoagulants (DOACs): state-of-the-art. Semin Thromb Hemost 2019;45(05):490-501

19 Lippi G, Franchini M, Favaloro EJ. Pharmacogenetics of vitamin K antagonists: useful or hype? Clin Chem Lab Med 2009;47(05): 503-515

20 Baluwala I, Favaloro EJ, Pasalic L. Therapeutic monitoring of unfractionated heparin - trials and tribulations. Expert Rev Hematol 2017;10(07):595-605

21 McCaughan GJB, Favaloro EJ, Pasalic L, Curnow J. Anticoagulation at the extremes of body weight: choices and dosing. Expert Rev Hematol 2018;11(10):817-828

22 Gosselin RC, Adcock DM, Bates SM, et al. International Council for Standardization in Haematology (ICSH) recommendations for laboratory measurement of direct oral anticoagulants. Thromb Haemost 2018;118(03):437-450

23 Lippi G, Favaloro EJ. Recent guidelines and recommendations for laboratory assessment of the direct oral anticoagulants (DOACs): is there consensus? Clin Chem Lab Med 2015;53 (02):185-197

24 DeLoughery E, Olson SR, McCarty OJT, Shatzel JJ. The safety and efficacy of novel agents targeting factor XI and XII in early phase human trials. Semin Thromb Hemost 2019;45(05):502-508

25 Horstman LL, McCauley RF, Jy W, Ahn YS. Tissue factor-negative cell-derived microparticles play a distinctive role in hemostasis: a viewpoint review. Semin Thromb Hemost 2019;45(05): 509-513

26 de Mello C, Loures G, Figueiredo Duarte RC, et al. Hemostatic abnormalities in dementia: a systematic review and meta-analysis. Semin Thromb Hemost 2019;45(05):514-522

27 Tabibian S, Shiravand Y, Shams M, et al. A comprehensive overview of coagulation factor $\mathrm{V}$ and congenital factor $\mathrm{V}$ deficiency. Semin Thromb Hemost 2019;45(05):523-543

28 Lippi G, Danese E, Favaloro EJ. Vascular disease and dementia: lipoprotein(a) as a neglected link. Semin Thromb Hemost 2019;45 (05):544-547

29 Zuo H, Yang F, Hu Y. Investigation of possible herb - drug interactions for the treatment of cardiovascular diseases. Semin Thromb Hemost 2019;45(05):548-551

30 McEwen BJ. Comment on "Investigation of possible herb - drug interactions for the treatment of cardiovascular diseases". Semin Thromb Hemost 2019;45(05):552-553

31 Ruiz-Argüelles GJ. Comment on: Inherited thrombophilia and pregnancy complications: should we test? Semin Thromb Hemost 2019;45(05):554-554

32 Arachchillage DRJ, Makris M. Reply to comment on: Inherited thrombophilia and pregnancy complications: Should we test? Semin Thromb Hemost 2019;45(05):555-556 